\title{
Pulmonary Manifestations among Patients with Primary Biliary Cirrhosis
}

\author{
Deniz Koksal*1, Aydin Seref Koksal ${ }^{2,3}$ and Ahmet Gurakar ${ }^{3}$ \\ ${ }^{1}$ Department of Chest Diseases, Hacettepe University School of Medicine, Ankara, Turkey; ${ }^{2}$ Department of Gastroenterology, \\ Sakarya University School of Medicine, Sakarya, Turkey; ${ }^{3}$ Division of Gastroenterology and Hepatology, Department of Medicine,
} Johns Hopkins School of Medicine, Baltimore, Maryland, USA

\begin{abstract}
Primary biliary cirrhosis (PBC) is a chronic progressive cholestatic liver disease caused by diffuse inflammation, destruction and fibrosis of the intrahepatic bile ducts, ultimately leading to cirrhosis, portal hypertension and liver failure. The pathogenesis of PBC is incompletely understood, but current data suggest roles for genetic susceptibility and environmental factors. PBC is often thought of as an organ-specific autoimmune disease, which mainly targets the liver; however, lung tissue is also a site for autoimmune involvement of PBC. The pulmonary manifestations of PBC include abnormalities in gas transfer and pulmonary function, subclinical alveolitis, interstitial lung disease, granulomatous lung disease, airway disease, pulmonary hypertension, pulmonary hemorrhage and pleural effusion.

(C) 2016 The Second Affiliated Hospital of Chongqing Medical University. Published by XIA \& HE Publishing Inc. All rights reserved.
\end{abstract}

\section{Introduction}

Primary biliary cirrhosis (PBC) is a chronic progressive cholestatic liver disease caused by diffuse inflammation, destruction and fibrosis of intrahepatic bile ducts, ultimately leading to cirrhosis, portal hypertension and liver failure. The typical patient is a middle-aged woman, presenting with fatigue and itching or asymptomatic with an unexplained hepatomegaly or elevated serum alkaline phosphatase level. ${ }^{1,2}$ Anti-mitochondrial antibodies (AMAs) are detected in more than $95 \%$ of the patients and are considered as a diagnostic hallmark of PBC.

The pathogenesis of PBC is incompletely understood. Current data suggest that it is an autoimmune disease and involves genetic susceptibility and environmental factors. ${ }^{3,4}$

Keywords: Primary biliary cirrhosis; Pulmonary involvement; Pulmonary functions.

Abbreviations: PBC, primary biliary cirrhosis; AMA, anti-mitochondrial antibodies; CTD, connective tissue diseases; SS, sjögren's syndrome; CREST, calcinosis, raynaud phenomenon, esophageal dysmotility, sclerodactyly, and telangiectasia BAL, bronchoalveolar lavage; ILD, interstitial lung disease; LIP, lymphoid interstitial pneumonia; NSIP, non-specific interstitial pneumonia; BOOP, bronchiolitis obliterans with organizing pneumonia; GLD, granulomatous lung disease. Received: 07 July 2016; Revised: 30 July 2016; Accepted: 31 July 2016

DOI: $10.14218 / \mathrm{JCTH} .2016 .00024$

*Correspondence to: Deniz Koksal, Department of Chest Diseases, Hacettepe University School of Medicine, Sihhiye Altindag, Ankara 06100, Turkey. Tel: +90532-4653980, Fax: +90-312-3100809, E-mail: dckoksal@gmail.com
It is more common in individuals with a family history of PBC and often associated with other autoimmune diseases. Autoimmune diseases can be seen in up to $84 \%$ of PBC patients, with $41 \%$ having more than one concomitant autoimmune disease. ${ }^{5,6}$ In a recent population-based cohort study, among 322 Chinese patients with PBC, 46.6\% had one or more connective tissue diseases (CTDs). The most common CTD was Sjögren's syndrome (SS) (36.2\%), followed by systemic lupus erythematosus $(3.7 \%)$, polymyositis $(3.1 \%)$, progressive systemic sclerosis $(2.8 \%)$ and rheumatoid arthritis $(2.8 \%){ }^{7}$

$\mathrm{PBC}$ is often thought of as an organ-specific autoimmune disease, which mainly targets the liver. However, lung tissue is also a possible site for autoimmune involvement of PBC and there is accumulating evidence indicating that PBC may involve multiple systems, including the pulmonary system. ${ }^{8}$ Therefore, physicians should be aware of potential pulmonary manifestations. Although the diagnosis of liver disease usually precedes pulmonary manifestations, the reverse can also occur. ${ }^{9}$ Sometimes, concomitant autoimmune diseases, which may be a contributor or primary etiology of a clinically apparent pulmonary disease, makes it difficult to discriminate PBC-associated lung involvement and other autoimmune diseases. ${ }^{10}$

The aim of this manuscript is to review pulmonary manifestations of PBC (Table 1). The pulmonary manifestations of PBC include abnormalities in gas transfer and pulmonary function, subclinical alveolitis, interstitial lung disease, granulomatous lung disease, airway disease, pulmonary hypertension, pulmonary hemorrhage and pleural effusion. Despite the main target of pulmonary involvement being the pulmonary parenchyma, the airways, pulmonary vasculature and pleura are also affected rarely. In the current literature, there is not much data about the pulmonary manifestations that occur among patients with PBC. Most of the literature has included small case series, case reports and scarce postmortem studies. Apart from this, it is difficult to discriminate sole pulmonary involvement of PBC from associated clinical conditions such as CTD-associated interstitial lung disease or portal hypertension related pulmonary hypertension. For these reasons, the frequency of pulmonary involvement in $\mathrm{PBC}$ is not known exactly.

\section{Gas transfer and pulmonary function abnormalities}

Gas transfer abnormalities can be investigated by measuring diffusing capacity for carbon monoxide. Subclinical lung impairment, mostly represented by a reduced diffusion of 
Table 1. Pulmonary manifestations of primary biliary cirrhosis

\begin{tabular}{ll}
\hline 1) Gas transfer abnormalities & \\
2) Subclinical alveolitis & $\begin{array}{l}\text { - Pulmonary fibrosis } \\
\text { 3) Interstitial } \\
\text { lung disease }\end{array}$ \\
$\begin{array}{l}\text { - Nonsphoid interstitial } \\
\text { pneumonia } \\
\text { Bronchiolitis obliterans } \\
\text { with organizing } \\
\text { pneumonia }\end{array}$ \\
4) Granulomatous lung \\
5) Airway disease
\end{tabular}

alveolar gasses, is a recognized complication of PBC. ${ }^{11,12}$ Rodriguez-Roisin et al. ${ }^{13}$ were the first to study lung function tests in patients with PBC. They compared 4 subgroups, all of which were non-smokers matched by age, sex, height and socioeconomic status. The groups included 7 sole PBC patients, 7 concurrent PBC and SS patients, 7 sole sicca complex patients, and 14 healthy controls. There was no significant difference in the mean pulmonary function test values, but the mean diffusion capacity for carbon monoxide was significantly reduced in those PBC patients with concurrent PBC and SS or with sole sicca complex. The authors concluded that the respiratory, clinical and functional abnormalities found in PBC were related to the presence of an associated SS. ${ }^{13}$ Later on, Uddenfeldt et al. ${ }^{14}$ investigated lung function abnormalities in a small number of patients with PBC $(n=25)$ and in age- and sex-matched healthy controls $(n=17)$. The PBC group was divided into two groups: symptomatic $(n=18)$ and asymptomatic $(n=7)$. The prevalence of lung function impairment was $56 \%$. Almost all abnormal lung function data were found in the symptomatic (i.e. pruritis, xanthoma, xanthelasmata, jaundice, hyperpigmentation, hepatosplenomegaly) patients. In the symptomatic PBC patients, there was a mean reduction in diffusion capacity and an elevated residual volume suggestive of hyperinflation, compared to the control group of patients. The authors suggested that PBC might be associated with a distal airway obstruction. However, they did not control the groups for smoking history or consider the presence of hepatopulmonary syndrome, which can cause a reduced diffusion in symptomatic, advanced PBC patients. ${ }^{14}$ Krowka et al. ${ }^{11}$ investigated the relationship between hepatic and pulmonary functions in never-smoked patients with PBC. They studied 67 patients, of who 20 were transplant candidates, and determined a significant relationship between the histological stage, disease severity of PBC and the steady-state diffusion capacity. Progressive deterioration of diffusion capacity was found to be associated with increasing severity of PBC. There was no relationship found between pulmonary function and the presence of sicca complex, SS or the clinical manifestations of portal hypertension. ${ }^{11}$ In another study that included 61 patients with different stages of PBC, the diffusion capacity was reduced in $24(39 \%)$ of the patients. There was no significant relationship between diffusion capacity, advancement of liver disease and concomitant SS. However, reduced diffusion capacity showed a significant correlation with the presence of complete or incomplete scleroderma (also known as CREST syndrome, standing for calcinosis, Raynaud phenomenon, esophageal dysmotility, sclerodactyly, and telangiectasia) and with the presence of circulating anti-centromere antibodies. ${ }^{12}$

\section{Subclinical alveolitis}

PBC is characterized by continued assault of T lymphocytemediated attack on the small intralobular bile ducts, eventually leading to their gradual destruction. ${ }^{1,2}$ It is suggested that lymphocytic infiltration of PBC may not be limited to the liver but may also involve the lungs. ${ }^{15}$ In a study aiming to determine whether subclinical inflammatory alveolitis is associated with PBC, the authors compared the number and types of cells in bronchoalveolar lavage (BAL) fluid from patients with $\mathrm{PBC}$, patients with alcoholic cirrhosis, and healthy controls. They found a subclinical alveolar inflammation comprised of T lymphocytes, mainly of the CD4 type, and activated alveolar macrophages in a high proportion of patients with PBC. ${ }^{16}$ One of the limitations of that study was the concomitant presence of SS in 7 out of 12 patients with PBC. Since it has been reported that up to $44 \%$ of patients with SS have subclinical alveolitis, it was difficult to discriminate the exact etiology. ${ }^{17}$ Spiteri et al. ${ }^{18}$ also studied BALderived cellular inflammatory patterns among patients with sarcoidosis, patients with PBC but without clinical lung involvement, and healthy controls. All patients with pulmonary sarcoidosis had lymphocytic alveolitis, with a proportion of $29.2 \%$. Six out of 10 patients with PBC showed evidence of alveolitis, with an elevated lymphocyte count of $27.6 \%$. Upon comparison of the patients with PBC and sarcoidosis, an overlap was found in the CD4/CD8 lymphocyte ratio predominance (4.13 vs. 5.6). The authors realized that, despite normal findings from physical examination and chest $x$-ray, 5 of 6 patients with alveolitis on BAL had an evidence of interstitial lung disease on subsequent computed tomography of the thorax. ${ }^{18}$

\section{Interstitial lung disease (ILD)}

PBC has been reported as associated with various ILDs, such as pulmonary fibrosis, lymphoid interstitial pneumonia (LIP), non-specific interstitial pneumonia (NSIP) and bronchiolitis obliterans with organizing pneumonia (BOOP). ${ }^{10,19}$ Concomitant SS increases the risk of ILD in patients with PBC. ${ }^{20}$ In a study composed of 109 consecutive PBC cases, concomitant SS was seen in 46 patients $(42.2 \%)$. While the frequency of ILD was $21.7 \%$ in PBC patients with SS, it was only $1.6 \%$ in those without SS. ${ }^{21}$

The findings of both radiographic interstitial pattern and restrictive ventilatory impairment with gas transfer defects in some of the patients with chronic active hepatitis and PBC has led some authors to suggest an association of pulmonary fibrosis with chronic liver disease. ${ }^{22-24}$ Although the prevalence of pulmonary fibrosis was poorly defined, one review estimated it to be less than $5 \% .{ }^{25}$ One of the reasons for this uncertainty could be the presence of concomitant CTDs, which would cloud the assumption of sole PBC attributable pulmonary fibrosis. ${ }^{10}$ In a necropsy series of 120 patients with PBC, none of the cases demonstrated pulmonary fibrosis findings. ${ }^{26}$ Golding et al. ${ }^{24}$ reported that $9(13 \%)$ out of 70 patients with 
PBC had both radiological and pulmonary functional changes suggestive of pulmonary fibrosis; however, only 2 of them had biopsy-proven pulmonary fibrosis. The histological variant of fibrosis in association with PBC was believed to be similar to usual interstitial pneumonitis. ${ }^{8,10,27}$ Shen et al. ${ }^{28}$ systematically addressed the development of ILD as a complication of PBC in a prospective study. The study included 178 consecutive PBC patients who were followed for 6 years. The incidence of ILD was $15.7 \%(n=28)$. Of the patients with ILD, $53.6 \%$ had pulmonary symptoms and $88.2 \%$ had diffusion and restrictive ventilation impairment on pulmonary function tests. Although $42.9 \%$ of the patients with ILD did not have any other CTD, presence of Raynoud phenomenon and association with other CTDs were the risk factors for PBC patients to develop ILD.

LIP is obviously associated with PBC and SS. Up to $25 \%$ of LIP cases occur in association with SS, and complete or incomplete SS can be noted in $84 \%$ of PBC patients. ${ }^{6,29}$ Shen et $a .^{28}$ performed lung biopsies in 5 patients with ILD. Histopathologic investigations revealed interstitial infiltrations with mainly lymphocytes suggestive of LIP in 3 patients. The other 2 biopsies were compatible with interstitial fibrosis, vascular hyperplasia and thickened vascular walls. ${ }^{28}$

BOOP may be one of the non-hepatic complications of PBC, especially in patients associated with other CTDs. ${ }^{30}$ Davison and Epstein ${ }^{31}$ reported a case of relapsing organizing pneumonitis in a man with PBC, CREST syndrome and chronic pancreatitis. BOOP can also manifest in isolated PBC cases. Chest radiographs show multiple, bilateral, peripherally distributed interstitial opacities. BOOP must be considered in the differential diagnosis of unresolved pneumonia in patients with PBC and respiratory symptoms. Strobel et al. ${ }^{32}$ reported an interesting case of PBC, for which the patient's open lung biopsy revealed BOOP, LIP and destructive bronchiolitis. This dual histopathology was also reflected in a bimodal clinical phase and gave a viewpoint about the therapy. The first phase was probably BOOP characterized by dramatic response to corticosteroid treatment (within a 1-week period) and the second phase was probably LIP characterized by a slow normalization (over a 1-year period). All recognizable signs of lung disease slowly and completely resolved after addition of azathioprine to the therapeutic regimen.

Data about the management of pulmonary involvement in the course of PBC is very limited. Review of the literature revealed that patients experience a widely variable response to therapeutic agents such as steroids, cyclosporine and azathioprine. ${ }^{10}$ This heterogenicity might be due to different histopathological patterns that could not be clinically distinguishable. The case presented by Strobel et $a^{32}$ also supported this hypothesis.

\section{Granulomatous lung disease (GLD)}

Sarcoidosis is a granulomatous disorder of unknown etiology that characteristically involves any organ of the body, but mainly the lungs. Although sarcoidosis does not meet the criteria for an autoimmune disorder, it can coexist with a wide variety of autoimmune diseases. ${ }^{33}$ There is also an association reported between PBC and sarcoidosis. ${ }^{34-36}$ A number of patients have features of both diseases, raising the question of whether they are within the spectrum of one disease or are two distinct clinical disorders that may share an autoimmune pathogenesis with some overlapping symptoms. ${ }^{37}$ Kishor et al. ${ }^{38}$ reviewed 17 patients with sarcoidosis and $\mathrm{PBC}$ and suggested that a common pathway contributes to granuloma formation in both disorders. The prevalence of GLD related to $\mathrm{PBC}$ is not known, but it seems to be rare based upon case reports and case series. A large necropsy series of 120 PBC patients reported only 2 patients $(1.6 \%)$ with widespread intrapulmonary granulomas without fibrosis; one of these patients had interstitial pattern on chest radiography and impaired gas transfer. ${ }^{26}$ Wallace et al. ${ }^{8}$ described 4 patients with PBC and concomitant SS, in whom symptomatic pulmonary involvement developed; three of these patients died of respiratory failure. The types of pulmonary involvement were interstitial fibrosis with accompanying vasculitis, which was thought to be a variant of idiopathic pulmonary fibrosis, lymphocytic bronchiolitis and GLD. GLD seems to be a steroid-responsive condition. Apart from steroids, other immunosuppressive drugs can also be used. ${ }^{39}$

\section{Airway disease}

Airflow obstruction has occasionally been reported in patients with PBC. ${ }^{11,14}$ Mild airway obstruction was present in 4 patients with concomitant sicca syndrome in a series of 67 patients with $\mathrm{PBC}^{8}{ }^{8}$ Obstructive lung disease was also reported in a patient with PBC and SS, secondary to an autoimmune process documented by immunofluorescent staining of lung parenchyma. ${ }^{8}$ Lymphocytic bronchitis/bronchiolitis is a non-specific type of airway disease characterized by infiltration of the large and small airways by lymphocytes. It was also described in a patient with $\mathrm{PBC}$, suggesting that lymphocytic bronchitis/bronchiolitis may be part of a generalized autoimmune process in PBC. ${ }^{40}$

\section{Pulmonary hypertension}

Pulmonary vascular abnormalities have been noted in the course of PBC. There is a well-recognized association between portal hypertension of cirrhosis and an increased incidence of pulmonary hypertension. ${ }^{41}$ In patients with liver disease, portal hypertension plays an important role in the pathogenesis of pulmonary hypertension, namely porto-pulmonary hypertension. ${ }^{42}$ In a prospective study including 178 consecutive PBC patients with a follow-up of 6 years, the incidence of pulmonary hypertension was $11.8 \%(n=21)$. Among these patients, 4 had moderate to severe pulmonary hypertension, and 1 patient died of right heart failure, indicating a poor prognosis. Pulmonary hypertension was closely associated with portal hypertension and immunological dysregulation. It was noteworthy that more than $30 \%$ of the patients with pulmonary hypertension did not show any evidence of portal hypertension, suggesting that other mechanisms may also be involved in the pathogenesis of pulmonary hypertension in PBC patients. ${ }^{43}$ However, there are a few case reports with PBC-associated pulmonary hypertension concomitant with normal portal venous pressure. ${ }^{44-46}$ This rarity might be due to dismissal of primary pulmonary hypertension since most of the patients had associated portal hypertension due to liver disease.

\section{Pulmonary hemorrhage}

Diffuse alveolar hemorrhage associated with PBC has been reported in 2 case reports. ${ }^{47,48}$ However, in both cases, there are some conflicting data for an associating pulmonary hemorrhage directly to PBC itself. Bissuel et al. ${ }^{47}$ reported a 
41-year-old woman presenting with life threatening intrapulmonary hemorrhages. The presence of antineutrophil cytoplasmic antibodies, focal segmental glomerulonephritis, and cutaneous leukocytoclastic vasculitis were all suggestive for the presence of a systemic vasculitis rather than PBCassociated alveolar hemorrhage. ${ }^{47}$ Komatsu et al. ${ }^{48}$ reported a patient with both PBC and Goodpasture's syndrome. Postmortem examination of both lungs revealed diffuse hemorrhage probably due to pulmonary vasculitis related to the Goodpasture's syndrome. ${ }^{48}$

\section{Pleural effusion}

Hepatic hydrothorax is defined as a pleural effusion in patients with cirrhosis, without a primary cardiac or pulmonary disease. The estimated prevalence of hepatic hydrothorax is about $5-10 \%$ in patients with cirrhosis. ${ }^{49,50}$ There are only a few case reports of PBC-associated hepatic hydrothorax. ${ }^{51,52}$ Other than hepatic hydrothorax, pleural effusion was described in a patient with LIP. Lymphocytic infiltration was shown in the thoracoscopic biopsy of the visceral pleura of the patient. ${ }^{53}$

\section{Conclusions}

In conclusion, $\mathrm{PBC}$ is a chronic progressive cholestatic liver disease caused by diffuse inflammation, destruction and fibrosis of intrahepatic bile ducts, ultimately leading to cirrhosis, portal hypertension and liver failure. The pathogenesis of PBC is incompletely understood. Current data suggest that it is an autoimmune disease involving genetic susceptibility and environmental factors. It is more common in individuals with a family history of PBC and often associated with other autoimmune diseases. PBC is often thought of as an organspecific autoimmune disease, which mainly targets the liver. However, lung tissue is also a site for autoimmune involvement of PBC. The main target of pulmonary involvement is the pulmonary parenchyma, but the airways, pulmonary vasculature and the pleura can also be affected rarely. Currently, the frequency of pulmonary involvement in PBC is not known exactly and there is no data for the presence of autoantibodies to diagnose PBC-related pulmonary involvement.

\section{Conflict of interest}

None

\section{Author contributions}

Writing content and formatting the table in the manuscript (DK), drafting sections of the document (ASK), critical revision and administration during manuscript writing (AG).

\section{References}

[1] Selmi C, Bowlus CL, Gershwin ME, Coppel RL. Primary biliary cirrhosis. Lancet 2011;377:1600-1609. doi: 10.1016/S0140-6736(10)61965-4.

[2] Kaplan MM, Gershwin ME. Primary biliary cirrhosis. N Engl J Med 2005;353: 1261-1273. doi: 10.1056/NEJMra043898.

[3] Jones DE. Pathogenesis of primary biliary cirrhosis. J Hepatol 2003;39:639648. doi: 10.1016/S0168-8278(03)00270-8.

[4] Gershwin ME, Selmi C, Worman HJ, Gold EB, Watnik M, Utts J, et al. Risk factors and comorbidities in primary biliary cirrhosis: a controlled interviewbased study of 1032 patients. Hepatology 2005;42:1194-1202. doi: 10.1002/ hep.20907.
[5] Strassburg CP, Manns MP. Autoimmune tests in primary biliary cirrhosis. Baillieres Best Pract Res Clin Gastroenterol 2000;14:585-599. doi: 10.1053/ bega.2000.0105.

[6] Culp KS, Fleming CR, Duffy J, Baldus WP, Dickson ER. Autoimmune associations in primary biliary cirrhosis. Mayo Clin Proc 1982;57:365-370.

[7] Wang L, Zhang FC, Chen $H$, Zhang X, Xu D, Li YZ, et al. Connective tissue diseases in primary biliary cirrhosis: a population-based cohort study. World J Gastroenterol 2013;19:5131-5137. doi: 10.3748/wjg.v19.i31.5131.

[8] Wallace JG Jr, Tong MJ, Ueki BH, Quismorio FP. Pulmonary involvement in primary biliary cirrhosis. J Clin Gastroenterol 1987;9:431-435. doi: 10.1097/ 00004836-198708000-00015.

[9] Martusewicz-Boros MM, Boros PW, Wiatr E. Respiratory system involvement in chronic liver diseases. Pol Arch Med Wewn 2013;123:635-642.

[10] Allan PF, Powers CR, Morris MJ. Pulmonary manifestations of primary autoimmune hepatobiliary disease. Clin Pulm Med 2005;12:232-245. doi: 10. 1097/01.cpm.0000171500.70809.d1.

[11] Krowka MJ, Grambsch PM, Edell ES, Cortese DA, Dickson ER. Primary biliary cirrhosis: relation between hepatic function and pulmonary function in patients who never smoked. Hepatology 1991;13:1095-1100. doi: 10 . 1002/hep.1840130615.

[12] Costa C, Sambataro A, Baldi S, Modena V, Todros L, Libertucci D, et al. Primary biliary cirrhosis: lung involvement. Liver 1995;15:196-201. doi: 10.1111/j.1600-0676.1995.tb00670.x.

[13] Rodriguez-Roisin R, Pares A, Bruguera M, Coll J, Picado C, Agusti-Vidal A, et al. Pulmonary involvement in primary biliary cirrhosis. Thorax $1981 ; 36$ : 208-212. doi: 10.1136/thx.36.3.208.

[14] Uddenfeldt $P$, Bjerle $P$, Danielsson A, Nyström L, Stjernberg N. Lung function abnormalities in patients with primary biliary cirrhosis. Acta Med Scand 1988;223:549-555. doi: 10.1111/j.0954-6820.1988.tb17694.x.

[15] Musialik J, Petelenz M, Jastrzebski D, Ziora D, Kondera-Anasz Z, Mertas A, et al. Lymphocyte subsets in peripheral blood and bronchoalveolar lavage in patients with primary biliary cirrhosis. Med Sci Monit 2001;7 suppl 1: 311-315.

[16] Wallaert B, Bonniere P, Prin L, Cortot A, Tonnel AB, Voisin C. Primary biliary cirrhosis. Subclinical inflammatory alveolitis in patients with normal chest roentgenograms. Chest 1986;90:842-848. doi: 10.1378/chest.90.6.842.

[17] Wallaert B, Dugas M, Dansin E, Perez T, Marquette CH, Ramon P, et al. Subclinical alveolitis in immunological systemic disorders. Transition between health and disease? Eur Respir J 1990;3:1206-1216.

[18] Spiteri MA, Johnson M, Epstein O, Sherlock S, Clarke SW, Poulter LW. Immunological features of lung lavage cells from patients with primary biliary cirrhosis may reflect those seen in pulmonary sarcoidosis. Gut 1990;31:208212. doi: 10.1136/gut.31.2.208.

[19] Spiteri MA, Clarke SW. The nature of latent pulmonary involvement in primary biliary cirrhosis. Sarcoidosis 1989;6:107-110.

[20] Chen CT, Tseng YC, Yang CW, Lin HH, Chen PJ, Huang TY, et al. Increased risks of spontaneous bacterial peritonitis and interstitial lung disease in primary biliary cirrhosis patients with concomitant Sjögren's syndrome. Medicine (Baltimore) 2016;95:e2537. doi: 10.1097/MD.0000000000002537.

[21] Liu B, Zhang FC, Zhang ZL, Zhang W, Gao LX. Interstitial lung disease and Sjögren's syndrome in primary biliary cirrhosis: a causal or casual association? Clin Rheumatol 2008;27:1299-1306. doi: 10.1007/s10067-0080917-x.

[22] Turner-Warwick M. Fibrosing alveolitis and chronic liver disease. Q J Med 1968;37:133-149.

[23] Mason AM, McIllmurray MB, Golding PL, Hughes DT. Fibrosing alveolitis associated with renal tubular acidosis. Br Med J 1970;4:596-599. doi: 10.1136/ bmj.4.5735.596.

[24] Golding PL, Smith M, Williams R. Multisystem involvement in chronic liver disease. Studies on the incidence and pathogenesis. Am J Med 1973;55: 772-782. doi: 10.1016/0002-9343(73)90258-1.

[25] Talwalkar JA, Lindor KD. Primary biliary cirrhosis. Lancet 2003;362:53-61. doi: 10.1016/S0140-6736(03)13808-1.

[26] Stanley NN, Woodgate DJ. Mottled chest radiograph and gas transfer defect in chronic liver disease. Thorax 1972;27:315-323. doi: 10.1136/ thx.27.3.315.

[27] Osaka M, Aramaki T, Okumura H, Kawanami O. Primary biliary cirrhosis with fibrosing alveolitis. Gastroenterol Jpn 1988;23:457-460.

[28] Shen M, Zhang F, Zhang X. Primary biliary cirrhosis complicated with interstitial lung disease: a prospective study in 178 patients. J Clin Gastroenterol 2009;43:676-679. doi: 10.1097/MCG.0b013e31818aa11e.

[29] Swigris J], Berry G], Raffin TA, Kuschner WG. Lymphoid interstitial pneumonia: a narrative review. Chest 2002;122:2150-2164. doi: $10.1378 /$ chest. 122.6.2150.

[30] Harada M, Hashimoto O, Kumemura H, Taniguchi E, Shiratsuchi M, Harada R, et al. Bronchiolitis obliterans organizing pneumonia in a patient with primary biliary cirrhosis and rheumatoid arthritis treated with prednisolone. Hepatol Res 2002;23:301. doi: 10.1016/S1386-6346(02)00006-2. 
[31] Davison AG, Epstein O. Relapsing organising pneumonitis in a man with primary biliary cirrhosis, CREST syndrome, and chronic pancreatitis. Thorax 1983;38:316-317. doi: 10.1136/thx.38.4.316.

[32] Strobel ES, Bonnet RB, Werner P, Schaefer HE, Peter HH. Bronchiolitis obliterans organizing pneumonia and primary biliary cirrhosis-like lung involvement in a patient with primary biliary cirrhosis. Clin Rheumatol 1998;17: 246-249. doi: 10.1007/BF01451058.

[33] Sakamoto N, Ishimatsu Y, Kakugawa T, Hara A, Hara S, Amenomori M, et al. Sarcoidosis in a patient with systemic sclerosis and primary biliary cirrhosis. Intern Med 2010;49:1609-1611. doi: 10.2169/internalmedicine.49.3457.

[34] Stanley NN, Fox RA, Whimster WF, Sherlock S, James DG. Primary biliary cirrhosis or sarcoidosis - or both. N Engl J Med 1972;287:1282-1284. doi: 10.1056/NEJM197212212872507.

[35] Maddrey WC. Sarcoidosis and primary biliary cirrhosis. Associated disorders? N Engl J Med 1983;308:588-590. doi: 10.1056/NEJM198303103081011.

[36] Fagan EA, Moore-Gillon JC, Turner-Warwick M. Multiorgan granulomas and mitochondrial antibodies. N Engl J Med 1983;308:572-575. doi: 10.1056/ NEJM198303103081006.

[37] Reilly J], Mark EJ. Case records of the Massachusetts General Hospital. Weekly clinicopathological exercises. Case-14-1998. A 49-year-old woman with primary biliary cirrhosis, pulmonary opacities, and a pleural eddusion. N Engl J Med 1998;338:1293-1301. doi: 10.1056/NEJM199804303381809.

[38] Kishor S, Turner MI, Borg BB, Kleiner DE, Cowen EW. Cutaneous sarcoidosis and primary biliary cirrhosis. J Am Acad Dermatol 2008;58:326-335. doi: 10.1016/j.jaad.2007.07.031.

[39] Franco I, Dubini A, Piciucchi S, Casoni G, Poletti V. Interstitial lung disease preceding primary biliary cirrhosis in a male patient. Rev Port Pneumol 2015 ; 21:214-217. doi: 10.1016/j.rppnen.2015.02.008.

[40] Chatté G, Streichenberger N, Boillot O, Gille D, Loire R, Cordier JF. Lymphocytic bronchitis/bronchiolitis in a patient with primary biliary cirrhosis. Eur Respir ] 1995;8:176-179. doi: 10.1183/09031936.95.08010176.

[41] Humbert M, Nunes $H$, Sitbon O, Parent F, Hervé P, Simonneau G. Risk factors for pulmonary arterial hypertension. Clin Chest Med 2001;22:459-475. doi: $10.1016 / \mathrm{S} 0272-5231(05) 70284-7$
[42] Yang YY, Lin HC, Lee WC, Hou MC, Lee FY, Chang FY, et al. Portopulmonary hypertension: distinctive hemodynamic and clinical manifestations. J Gastroenterol 2001;36:181-186. doi: 10.1007/s005350170126.

[43] Wallwork J, Williams R, Calne RY. Transplantation of liver, heart, and lungs for primary biliary cirrhosis and primary pulmonary hypertension. Lancet 1987; 2:182-185. doi: 10.1016/S0140-6736(87)90765-3.

[44] Yoshida EM, Erb SR, Ostrow DN, Ricci DR, Scudamore CH, Fradet G. Pulmonary hypertension associated with primary biliary cirrhosis in the absence of portal hypertension: a case report. Gut 1994;35:280-282. doi: 10.1136/ gut.35.2.280.

[45] Shen M, Zhang F, Zhang X. Pulmonary hypertension in primary biliary cirrhosis: a prospective study in 178 patients. Scand J Gastroenterol 2009; 44:219-223. doi: 10.1080/00365520802400883.

[46] Honma F, Shio K, Monoe K, Kanno Y, Takahashi A, Yokokawa J, et al. Primary biliary cirrhosis complicated by polymyositis and pulmonary hypertension. Intern Med 2008;47:667-669. doi: 10.2169/internalmedicine.47.0793.

[47] Bissuel F, Bizollon T, Dijoud F, Bouletreau P, Cordier JF, Chazot C, et al. Pulmonary hemorrhage and glomerulonephritis in primary biliary cirrhosis. Hepatology 1992;16:1357-1361. doi: 10.1002/hep.1840160609.

[48] Komatsu T, Utsunomiya K, Oyaizu T. Goodpasture's syndrome associated with primary biliary cirrhosis. Intern Med 1998;37:611-613. doi: 10.2169/ internalmedicine.37.611.

[49] Lazaridis KN, Frank JW, Krowka MJ, Kamath PS. Hepatic hydrothorax: pathogenesis, diagnosis, and management. Am J Med 1999;107:262-267. doi: 10.1016/S0002-9343(99)00217-X.

[50] Strauss RM, Boyer TD. Hepatic hydrothorax. Semin Liver Dis 1997;17:227232. doi: $10.1055 / \mathrm{s}-2007-1007200$.

[51] Alhaji M, Sadikot RT. A 62-year-old female patient with left-sided pleural effusion. Expert Rev Respir Med 2013;7:455-458. doi: 10.1586/17476348. 2013.816567.

[52] Wojcikiewicz TG, Gupta S. Primary biliary cirrhosis presenting with ascites and a hepatic hydrothorax: a case report. J Med Case Rep 2009;3:7371. doi: 10.4076/1752-1947-3-7371.

[53] Weissman E, Becker NH. Interstitial lung disease in primary biliary cirrhosis. Am J Med Sci 1983;285:21-27. doi: 10.1097/00000441-198305000-00004. 